\title{
Insights into Persistent Toxic Substances in Protective Cases of Mobile Phones: Occurrence, Health Risks, and Implications
}

Qilu Li,* Meng Yuan, Jingfang Shangguan, Mengran Guo, Jiajia Wu, Ying Zhang, Mengyuan Pei, Ziyi Zhang, Mengqiong Zhang, Yujing Sun, Chongguo Tian, Jun Li, and Gan Zhang

Cite This: Environ. Sci. Technol. 2021, 55, 6076-6086

Read Online

ACCESS | Lill Metrics \& More | 国 Article Recommendations ｜ st Supporting Information

ABSTRACT: Despite the popularity of smartphones worldwide, persistent toxic substances (PTSs) in protective cases of mobile phones (PCMPs) and their health risks via direct skin contact have been ignored. This study investigated PTSs in PCMPs made in China with different materials and sales territory and their potential harm to human health. Polybrominated diphenyl ethers (PBDEs, $6.40 \mathrm{ng} / \mathrm{g}$ ), new brominated flame retardants (NBFRs, $144 \mathrm{ng} / \mathrm{g}$ ), organophosphate esters (OPEs, $10.1 \mu \mathrm{g} / \mathrm{g}$ ), short-chain chlorinated paraffins (SCCPs, $3.58 \mu \mathrm{g} / \mathrm{g}$ ), medium-chain chlorinated paraffins (MCCPs, $3.17 \mu \mathrm{g} / \mathrm{g}$ ), and heavy metals (HMs, $72.3 \mu \mathrm{g} / \mathrm{g}$ ) were detected. It was found that the different concentrations and

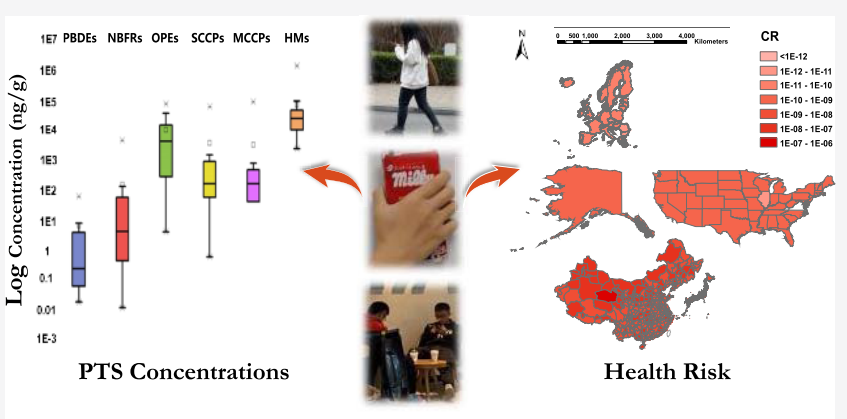

Protective Case of Mobile Phone compositions depend on the material, region, and use. Moreover, the raw materials used to fabricate PCMPs are of variable quality and may include recycled plastic waste. There are no standard quality specifications for PCMPs, and different materials have different properties, including specific surface area and adsorption ability. The risk assessment performed by Monte Carlo simulations indicated that the PTSs evaluated pose no health risks to the general population and may have adverse effects on individual high-exposure populations. According to the results of this work, it is suggested that more stringent global specifications for the selection of raw materials should be established, including the content and structural characteristics of PTSs, limitations on the use of additives in the production process, and the handling after use.

\section{INTRODUCTION}

With the rapid development of the electronics industry, ${ }^{1,2}$ smartphones have become popular worldwide, with 1.57 billion users in 2018 and an expected 3.8 billion by $2021 .^{3-5}$ Recent incidents causing personal injuries have drawn attention to the quality and safety of mobile phone accessories. $^{6}$ As one of the most commonly used mobile phone accessories, protective cases of mobile phones (PCMPs) are often in direct contact with the skin for long periods, but the potential health risks caused by the pollutants in PCMPs have been ignored.

Approximately $84 \%$ of PCMPs are made of plastic, ${ }^{7}$ these plastics may contain persistent toxic substances (PTSs), including brominated flame retardants (BFRs), ${ }^{8,9}$ organophosphate esters (OPEs), ${ }^{10}$ chlorinated paraffins (CPs), ${ }^{11}$ and heavy metals (HMs). ${ }^{12}$ BFRs include polybrominated diphenyl ethers (PBDEs) and new brominated flame retardants (NBFRs). Among them, up to $90 \%$ of commercial decabromodiphenyl ether (deca-BDE) and $70 \%$ of commercial octa-bromodiphenyl ether (octa-BDE) are ultimately used in plastics. ${ }^{13}$ OPEs and CPs are widely utilized as plasticizers in plastic products. ${ }^{11,14}$ PTSs can enter the body through various pathways when humans are in close contact with plastic products and cause central and peripheral nervous system damage, immune and reproductive system diseases, and even cancer. $^{15-18}$

Among smartphone users, $87 \%$ will use PCMPs, most of whom will use more than one PCMP over the service life of the phone. ${ }^{5}$ As a result, PCMPs have been dispersed throughout various regions of the country. Cross-border ecommerce has a positive impact on the annual growth of China's international trade, including the international PCMP trade. ${ }^{19}$ PCMPs made in China are increasingly being exported to the United States, Europe, Japan, and elsewhere. ${ }^{7}$ These international transactions have resulted in the global distribution of the PTSs contained in PCMPs.

Considering the potential adverse effects of PCMPs on human health and the environment, in this study, we determined (1) the characteristics of PTS pollution from PCMPs according to the material and trade type (domestic and export sales), (2) the influence of raw materials on PTS

Received: November 10, 2020

Revised: March 18, 2021

Accepted: April 1, 2021

Published: April 15, 2021

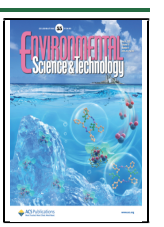


pollution characteristics, and (3) human health risks induced by PTS during PCMP use.

\section{MATERIALS AND METHODS}

Sample Collection. PCMP samples, manufactured in China, were mainly divided into two categories according to the trade type. One was for domestic sales and available in China (China-PCMP, CPCMP) and the other one was for export trade and available in the United Kingdom and the United States (UK/U.S.-PCMP, UPCMP). Since polycarbonate (PC), thermoplastic polyurethane (TPU), and silica gel (SG) account for $80 \%$ of the CPCMP market, ${ }^{7}$ the samples in this study were classified into the above three categories by different materials. Eighty-six of CPCMPs were bought from online and offline marketing in China in 2018, including 16 of PC, 12 of TPU, and 15 of SG. Ten of UPCMPs were bought from local supermarkets in the United States ( 3 of PC and 2 of SG) and the United Kingdom (2 of PC, 1 of SG, and 2 of TPU). A total of 43 volunteers were recruited to use CPCMPs for a 3 month comparative research, and two users for each type of PCMP. As the used UPCMPs were difficult to collect, in the PTS analysis of the used PCMPs, only the used CPCMPs were investigated.

Sample Extraction. The extract and cleanup methods have been described in detail in previous work. ${ }^{20-22}$ The PCMP samples were washed with deionized water and dried in a vacuum dryer. Each sample was cut into tiny fragments (approximately $3 \mathrm{~mm} \times 3 \mathrm{~mm}$ ) and mixed well. After wrapping with aluminum foil, these samples were placed in sealed bags and stored at $-20{ }^{\circ} \mathrm{C}$ until extraction. The full name and abbreviation of target pollutants (PBDEs, NBFRs, OPEs, short-chain chlorinated paraffins (SCCPs), medium-chain chlorinated paraffins (MCCPs), and HMs) are listed in the Supporting Information (SI, Table S1).

PBDEs, NBFRs, and OPEs. The above pretreated sample (1 g) was put into a $50 \mathrm{~mL}$ centrifuge tube with $30 \mathrm{~mL}$ of dichloromethane (DCM) and $n$-hexane ( $n$-hex) (volume ratio of $1: 1)$. Three pieces of $5 \mathrm{~mm} \times 5 \mathrm{~mm}$ copper and recovery indicators (20 ng of PCB198 and PCB209 for PBDEs and NBFRs; $20 \mathrm{ng}$ of $d_{12}$-TCEP, $d_{27}$-TnBP, and $d_{15}$-TPhP for OPEs) were added, and the sample was extracted by ultrasound three times, each time for $10 \mathrm{~min}$. The supernatant was transferred into a $100 \mathrm{~mL}$ round-bottom flask after each ultrasound extraction and then supplemented with $30 \mathrm{~mL}$ of a mixture solution of DCM and $n$-hex $(\mathrm{v} / \mathrm{v}, 1: 1)$. The extraction liquid was concentrated by a rotary evaporator to $1 \mathrm{~mL}$. After that, the liquid was purified, and fractionation was performed using chromatographic columns.

The first column, with $0.5 \mathrm{~g}$ of anhydrous sodium sulfate and $4 \mathrm{~g}$ of neutral silica gel from top to bottom, was prewashed with a mixture of DCM and $n$-hex $(\mathrm{v} / \mathrm{v}, 1: 1)$. Two fractions were obtained: Fraction 1 (F1) was eluted with $30 \mathrm{~mL}$ of $n$-hex and Fraction 2 (F2) containing OPEs was eluted with $25 \mathrm{~mL}$ of ethyl acetate. Then, after F1 was passed through the second column (from top to bottom: $0.5 \mathrm{~g}$ of anhydrous sodium sulfate, $4 \mathrm{~g}$ of sulfuric acid silica gel, $3 \mathrm{~g}$ of neutral silica gel, and $3 \mathrm{~g}$ of alumina), $35 \mathrm{~mL}$ of eluent was taken out as Fraction 3 (F3), which contained PBDEs and NBFRs. F2 and F3 were concentrated to $100 \mu \mathrm{L}$ of $n$-hex under a gentle flow of nitrogen. Finally, the samples were stored at $-20{ }^{\circ} \mathrm{C}$. Prior to analysis, $100 \mathrm{ng}$ of ${ }^{13} \mathrm{C}_{12}$-PCB141, $100 \mathrm{ng}$ of hexamethylbenzene, and $100 \mathrm{ng}$ of ${ }^{13} \mathrm{C}_{8}$-mirex were added as internal standards for PBDEs, NBFRs, and OPEs, respectively.
SCCPs and MCCPS. The fragment sample (1 g) was mixed with $20 \mathrm{ng}$ of ${ }^{13} \mathrm{C}$-trans-chlordane as the surrogate standard and Soxhlet-extracted with DCM for $48 \mathrm{~h}$. Three pieces of copper were added to remove elemental sulfur. The extract of each sample was concentrated by a rotary evaporator and then cleaned using a multilayer column that contained $1 \mathrm{~g}$ of anhydrous $\mathrm{Na}_{2} \mathrm{SO}_{4}, 2 \mathrm{~g}$ of Florisil, $3 \mathrm{~g}$ of neutral silica gel, and $3 \mathrm{~g}$ of neutral alumina from top to bottom. The eluent (30 $\mathrm{mL}$ ) was concentrated to approximately $25 \mu \mathrm{L}$ using a gentle stream of nitrogen gas. Before instrument analysis, $100 \mathrm{ng}$ of ${ }^{13} \mathrm{C}_{8}$-mirex was added as an internal standard.

HMs. The pretreated sample $(0.1 \mathrm{~g})$ was put into a poly(tetrafluoroethylene) digestion tank and $8 \mathrm{~mL}$ of nitric acid was added. The digestion tank was placed on an electric heating board, and the sample was predissolved at $130{ }^{\circ} \mathrm{C}$ for $40 \mathrm{~min}$ and cooled down to room temperature. Then, $2 \mathrm{~mL}$ of hydrofluoric acid and $2 \mathrm{~mL}$ of hydrogen peroxide were added to the solution. It was allowed to stand for $10 \mathrm{~min}$ and then digested. The digestion solution was acid-driven to $0.5 \mathrm{~mL}$ and then transferred to a $50 \mathrm{~mL}$ volumetric flask and diluted with nitric acid solution ( $\mathrm{pH}$ 3.0). Finally, the solution was purified using a $0.45 \mu \mathrm{m}$ polypropylene filter and stored at $-4{ }^{\circ} \mathrm{C}$.

Instrumental Analysis. The qualitative and quantitative identification of PBDEs, NBFRs, OPEs, SCCP, and MCCP congener groups was based on previously reported retention time, signal shape, and correct isotope ratio. ${ }^{20-22}$

PBDEs and NBFRs. Samples were analyzed by an Agilent gas chromatograph coupled to a triple quadrupole mass spectrometer (gas chromatography-mass spectrometry/tandem mass spectrometry (GC-MS/MS), 7890B-7000D) with a $\mathrm{BD}-5 \mathrm{HT}$ column (length $15 \mathrm{~m}$, inner diameter $0.25 \mu \mathrm{m}$, and liquid film thickness $0.1 \mu \mathrm{m}$ ). The injection volume was $2 \mu \mathrm{L}$ with splitless mode, and the injector temperature was $240{ }^{\circ} \mathrm{C}$. The initial oven temperature was set at $90{ }^{\circ} \mathrm{C}$ and held for 3 min, increased to $120^{\circ} \mathrm{C}$ and held for $1 \mathrm{~min}$, then increased to $240{ }^{\circ} \mathrm{C}$ at $12{ }^{\circ} \mathrm{C} / \mathrm{min}$ and held for $1 \mathrm{~min}$, and finally heated to $320^{\circ} \mathrm{C}$ at $40{ }^{\circ} \mathrm{C} / \mathrm{min}$ and held for $8 \mathrm{~min}$. The ion source was a negative chemical source, and the ion source temperature was $250{ }^{\circ} \mathrm{C}$.

OPEs. The OPEs were analyzed using an Agilent gas chromatograph coupled to a triple quadrupole mass spectrometer (GC-MS/MS, 7890B-7000D) with an HP-5MS capillary column (length $30 \mathrm{~m}$, inner diameter $0.25 \mathrm{~mm}$, and liquid film thickness $0.25 \mu \mathrm{m}$ ). The ion source was a highsensitivity electron impact ionization source. The injection volume was $2 \mu \mathrm{L}$ with pulsed splitless mode. The initial temperature was set at $50{ }^{\circ} \mathrm{C}$, increased to $70{ }^{\circ} \mathrm{C}$ and held for $2 \mathrm{~min}$, and then increased to $300^{\circ} \mathrm{C}$ at $20^{\circ} \mathrm{C} / \mathrm{min}$ and held for $6 \mathrm{~min}$. The inlet temperature was $230^{\circ} \mathrm{C}$, and the ion source temperature was $300{ }^{\circ} \mathrm{C}$.

SCCPs and MCCPs. The SCCPs and MCCPs were analyzed using an Agilent gas chromatograph coupled to a quadrupole time-of-flight mass spectrometer (GC/Q-TOF, 7250B) with an HP-5MS (length $30 \mathrm{~m}$, inner diameter $0.25 \mathrm{~mm}$, liquid film thickness $0.25 \mu \mathrm{m})$. The ion source was a negative chemical source. The injection volume was $2 \mu \mathrm{L}$ with splitless mode, and the constant flow injection mode was $1.0 \mathrm{~mL} / \mathrm{min}$. The rising column temperature program started with the initial temperature at $100{ }^{\circ} \mathrm{C}$ for $1 \mathrm{~min}$. After that, it was increased to $160^{\circ} \mathrm{C}$ at $5{ }^{\circ} \mathrm{C} / \mathrm{min}$ and held for $2 \mathrm{~min}$ and then increased to $310{ }^{\circ} \mathrm{C}$ at $30{ }^{\circ} \mathrm{C} / \mathrm{min}$ and held for $12 \mathrm{~min}$. The inlet temperature was $300{ }^{\circ} \mathrm{C}$, and the ion source temperature was $160^{\circ} \mathrm{C}$. 
HMs. HMs were analyzed by an Agilent inductively coupled plasma mass spectrometer (7900-G8403A). The radio frequency power was set at $1500 \mathrm{~W}$. The flow rates of atomization gas flow plasma gas, carrier gas, auxiliary gas, and helium gas were $15,0.80,0.40$, and $4.0 \mathrm{~L} / \mathrm{min}$, respectively. The plasma gas flow rate was $15 \mathrm{~L} / \mathrm{min}$. The temperature of the spray chamber was $2{ }^{\circ} \mathrm{C}$.

Quality Assurance and Quality Control (QA/QC). Before use, all glassware was washed with cleaning solution (RBS T 115, purchased from Chemical Products R. Borghgraef S.A.) and deionized water, then dried in an oven, and calcined in a muffle furnace at $450{ }^{\circ} \mathrm{C}$ for $8 \mathrm{~h}$. Recovery standard indicators were added to samples (Table S2), and the calculation method of the recovery rate is shown in the SI, Text 1. Procedural blank and spiked samples were pretreated and analyzed using the same method as that for PMCP samples to control the extraction and cleanup procedures. A procedural blank was run every 10 samples. The analyte levels of target compounds in the procedural blank were $4.17 \pm 3.02$ pg (PBDEs), $8.44 \pm 0.49$ pg (NBFRs), $11.6 \pm 7.90$ pg (OPEs), $18.0 \pm 13.1 \mathrm{pg}$ (SCCPs), $15.8 \pm 9.23 \mathrm{pg}$ (MCCPs), and $38.4 \pm 45.9 \mathrm{ng}$ (HMs). The recoveries in all spiked samples are listed in Table S3. The method detection limits (MDLs) were assigned from all of the blanks as the mean concentration plus three times the standard deviation (Table S2).

Human Exposure Model and Parameters. The health risks associated with PTS through dermal exposure pathways were assessed using the methods reported by the Environmental Protection Agency. ${ }^{23-26}$ Reference values for exposure factors in eqs $1-10$ are summarized in SI Text 2 and Tables S4-S6.

$$
\begin{aligned}
& \mathrm{ADD}_{1}=\frac{\mathrm{C} \times \mathrm{SA} \times \mathrm{ED} \times \mathrm{APC}}{\mathrm{BW}} \\
& \mathrm{ADD}_{2}=\frac{\mathrm{C} \times \mathrm{SA} \times \mathrm{AF} \times \mathrm{ABS} \times \mathrm{EF} \times \mathrm{ED}}{\mathrm{BW} \times \mathrm{AT}}
\end{aligned}
$$

where $\mathrm{ADD}_{1}$ is the daily exposure per unit weight of PBDEs, NBFRs, and OPEs (ng/kg Bwd) and $\mathrm{ADD}_{2}$ is the daily exposure per unit weight of HMs $(\mathrm{mg} / \mathrm{kg} \mathrm{Bwd})$.

$$
\begin{aligned}
& \mathrm{RfD}=\mathrm{RfD}_{\mathrm{o}} \times \mathrm{ABS}_{\mathrm{GI}} \\
& \mathrm{HI}=\sum \mathrm{HQ}=\sum \frac{\mathrm{ADD}}{\mathrm{RfD}} \\
& \mathrm{SF}=\frac{\mathrm{SFo}}{\mathrm{ABS}_{\mathrm{GI}}} \\
& \mathrm{CR}=\mathrm{SF} \times \mathrm{ADD}_{2}
\end{aligned}
$$

To assess the overall risk of noncarcinogenic effects exerted by more than one monomer or element, a Hazard Index (HI) approach has been applied. If $\mathrm{HI} \leq 1$, noncancerous effects are unlikely for human health, whereas for $\mathrm{HI}>1$, there might be possible adverse health effects. $\mathrm{HI}>10$ is considered to be a high chronic risk. Because the carcinogenic slope factor is available only for As, the carcinogenic risk (CR) of As was estimated, which indicates a safe acceptable scope when it is lower than a range of $1.0 \times 10^{-6}-1.0 \times 10^{-4}$.

$$
\mathrm{ADD}_{3}=\frac{\mathrm{C} \times \mathrm{SA} \times \mathrm{AF} \times \mathrm{ABS} \times \mathrm{Et}}{\mathrm{BW}}
$$

$$
\text { MOE }=\frac{\text { NOAEL }}{\mathrm{ADD}_{3}}
$$

$\mathrm{ADD}_{3}$ is the daily exposure per unit weight of SCCPs and MCCPs. The margin of exposure (MOE) is used to assess the severity of possible health risks posed by SCCPs and MCCPs where the no observed adverse effect level (NOAEL) values of SCCPs and MCCPs are 100 and $23 \mathrm{mg} / \mathrm{kg} /$ day, respectively, as stated in the European Union Risk Assessment Report. ${ }^{25} \mathrm{~A}$ total uncertainty factor of 1000 was applied. This included a factor of 10 for a research period of less than 1 year, a factor of 10 for cross-species differences, and a factor of 10 for individual differences. ${ }^{27}$ The MOE value greater than the uncertainty factor of 1000 indicates that no significant health risks were posed to people.

The spatial distribution of health risks was calculated using eqs 9 and 10 to indicate the noncarcinogenic risk and CR caused by PCMPs consumption in China's domestic and exported cities in the United States, European countries, and Japan prefecture in $2018 .^{28}$ China included prefecture-level cities in 23 provinces, 5 autonomous regions, and 4 municipalities other than Taiwan, Macao, and the Hong Kong Special Administrative Region.

$$
\begin{aligned}
\mathrm{HI}_{U} & =\sum_{u} \frac{\sum\left(\mathrm{ADD}_{j} / \mathrm{Rfd}_{j}\right) \times N_{U}}{\sum_{U} P} \\
\mathrm{IR}_{U} & =\sum_{u} \frac{\sum\left(\mathrm{ADD}_{j} \times \mathrm{SF}\right) \times N_{U}}{\sum_{U} P}
\end{aligned}
$$

Monte Carlo Simulation. PCMP samples were purchased at random, and there were complex and various factors in the process of exposure, which has led to the uncertainty of health risk assessment. ${ }^{26}$ The risk assessment was carried out by a Monte Carlo simulation to minimize the uncertainty. Oracle crystal ball (version 11.1.2.4) was used for the Monte Carlo simulation, which was used to establish a model for the data in this study and conduct 10000 simulations to assess the health risk of PTSs in PCMPs to humans. The average and 95th percentile of the probability density of HI were used for estimating the exposure risk of the general population and the high-exposure population, respectively. By analyzing the probability distribution of $\mathrm{HQ}$, the probability of the influence of PCMPs on the health of the general population and the high-exposure population could be obtained.

\section{RESULTS AND DISCUSSION}

The average concentrations of PBDEs, NBFRs, OPEs, SCCPs, MCCPs, and HMs in all PCMP samples were $6.40 \mathrm{ng} / \mathrm{g}, 144$ $\mathrm{ng} / \mathrm{g}, \quad 10.1 \mu \mathrm{g} / \mathrm{g}, \quad 3.58 \mu \mathrm{g} / \mathrm{g}, \quad 3.17 \mu \mathrm{g} / \mathrm{g}$, and $72.3 \mu \mathrm{g} / \mathrm{g}$, respectively. On comparing the concentration of PTSs in other studies, it is found that the concentration of PBDEs was much lower than the plastics retained on the South China Sea island $(22 \mu \mathrm{g} / \mathrm{g}){ }^{29}$ the concentration of OPEs was similar to the plastic decoration materials $(44.5 \mu \mathrm{g} / \mathrm{g}){ }^{30}$ The concentrations of SCCPs and MCCPs were lower than those in rubber track products $(3.64 \text { and } 41.4 \mathrm{mg} / \mathrm{g})^{15}$ but higher than those in Chinese plastic food packaging (188 and $644 \mathrm{ng} / \mathrm{g}){ }^{21}$ Table S7 lists the maximum value, minimum value, median value of concentrations, detection rate, etc. Among these six classes of contaminants, the highest relative mass fractions of each were BDE209 (28.0\%), DBDPE (88.1\%), TPP (70.6\%), $\mathrm{C}_{10}$ (34.5\%), $\mathrm{C}_{14}(70.6 \%)$, and $\mathrm{Zn}(56.1 \%)$. 

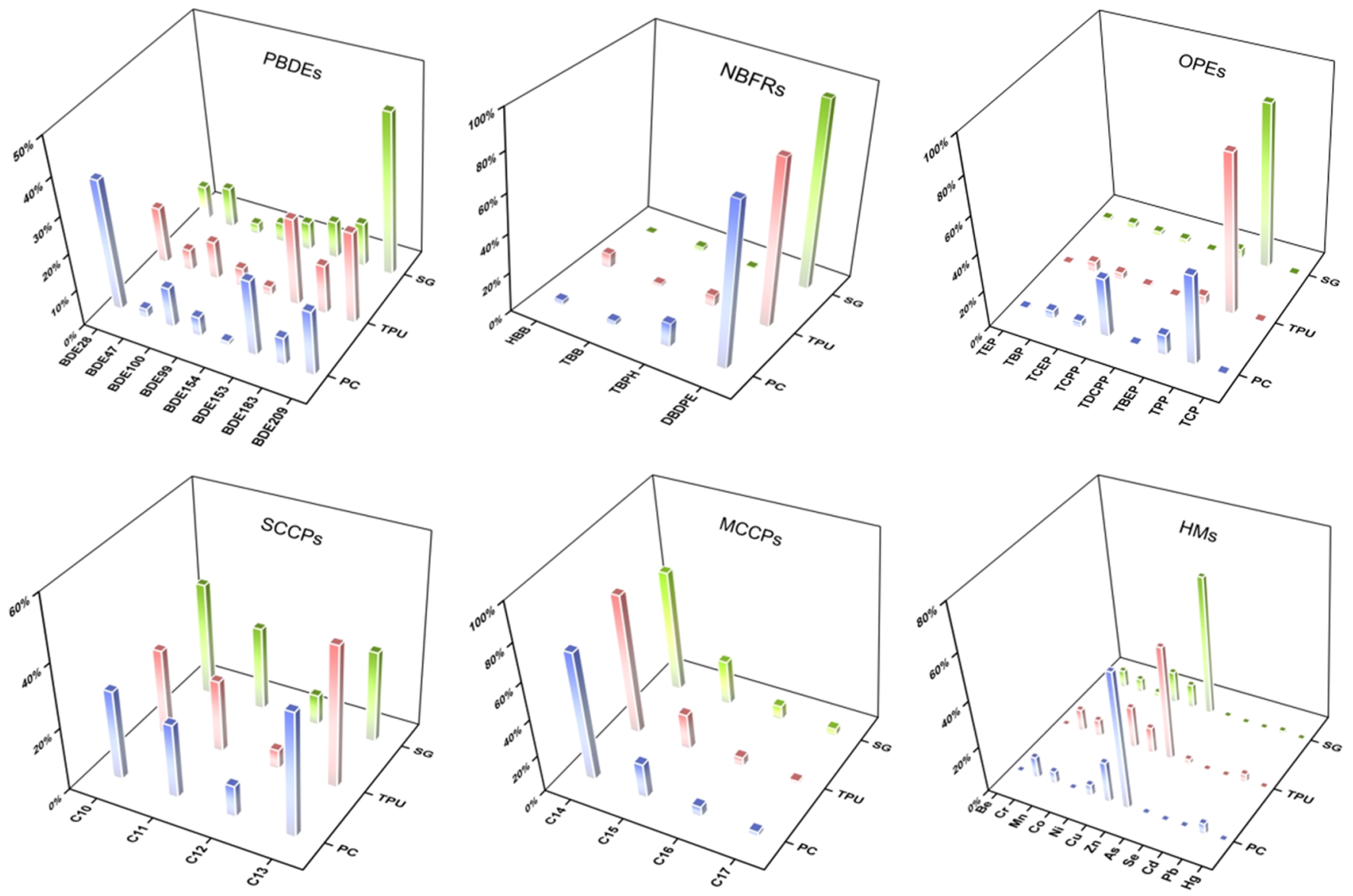

Figure 1. Composition of PTS in different PCMP materials.

PTS Concentrations in Different PCMP Materials. In PC, the respective PBDE, NBFR, OPE, SCCP, MCCP, and $\mathrm{HM}$ concentrations were $8.74 \mathrm{ng} / \mathrm{g}, 238 \mathrm{ng} / \mathrm{g}, 0.10 \mu \mathrm{g} / \mathrm{g}, 8.00$ $\mu \mathrm{g} / \mathrm{g}, 6.71 \mu \mathrm{g} / \mathrm{g}$, and $125 \mu \mathrm{g} / \mathrm{g}$. By contrast, the PBDE $(8.12$ and $2.33 \mathrm{ng} / \mathrm{g}$ ), NBFR (33.2 and $121 \mathrm{ng} / \mathrm{g}$ ), SCCP (0.62 and $0.38 \mu \mathrm{g} / \mathrm{g}$ ), MCCP $(0.14$ and $1.13 \mu \mathrm{g} / \mathrm{g}$ ), and HM (40.4 and $35.7 \mu \mathrm{g} / \mathrm{g}$ ) concentrations in TPU and SG were lower than those in PC. The OPE concentration was much lower in PC than in TPU $(19.0 \mu \mathrm{g} / \mathrm{g})$ and SG $(13.8 \mu \mathrm{g} / \mathrm{g})$. One-way analysis of variance (ANOVA) (Table S8) showed that the OPE concentration differed significantly $(P<0.05)$ between $\mathrm{PC}$ and the other two materials. Comparisons of the PTS concentrations in CPCMPs and UPCMPs are shown in Figure S1. The PBDE, NBFR, SCCP, and MCCP concentrations in PC were much higher in CPCMPs (11.4 ng/g, $295 \mathrm{ng} / \mathrm{g}, 10.5$ $\mu \mathrm{g} / \mathrm{g}$, and $8.78 \mu \mathrm{g} / \mathrm{g}$, respectively) than in UPCMPs $(0.25 \mathrm{ng} /$ $\mathrm{g}, 54.4 \mathrm{ng} / \mathrm{g}, 0.06 \mu \mathrm{g} / \mathrm{g}$, and $0.09 \mu \mathrm{g} / \mathrm{g}$, respectively), whereas the OPE concentration in PC was much lower in CPCMPs $(0.44 \mu \mathrm{g} / \mathrm{g})$ than in UPCMPs $(2.79 \mu \mathrm{g} / \mathrm{g})$. The BFR and MCCP concentrations in TPU were lower in CPCMPs (1.50 $\mathrm{ng} / \mathrm{g}$ and $0.13 \mu \mathrm{g} / \mathrm{g}$, respectively) than in UPCMPs (223 and $0.20 \mathrm{ng} / \mathrm{g}$, respectively), whereas the OPE concentration in TPU was much higher in CPCMPs $(22.1 \mu \mathrm{g} / \mathrm{g})$ than in UPCMPs $(0.03 \mu \mathrm{g} / \mathrm{g})$. In $\mathrm{SG}$, the PBDE and NBFR concentrations were higher in UPCMPs $(10.9 \mathrm{ng} / \mathrm{g}$ and 0.37 $\mu \mathrm{g} / \mathrm{g}$, respectively) than in CPCMPs $(0.62 \mathrm{ng} / \mathrm{g}$ and $0.07 \mu \mathrm{g} /$ g, respectively), whereas the OPE, SCCP, MCCP, and HM concentrations were much higher in CPCMPs (15.3, 0.45, 1.36 , and $40.9 \mu \mathrm{g} / \mathrm{g}$, respectively) than in UPCMPs (6.64, $0.03,0.05$, and $9.28 \mu \mathrm{g} / \mathrm{g}$, respectively). Table S9 shows the results of a paired $t$ test of concentrations for different materials of CPCMPs and UPCMPs. In CPCMPs, there were significant differences in the PBDE and SCCP concentrations in PC and SG and in the OPE concentrations in PC and the other materials $(P<0.05)$. In UPCMPs, there was a significant difference in the SCCP concentrations between PC and TPU $(P<0.05)$.

PTS Compositions in Different PCMP Materials. Figure 1 shows the PTS compositions of different materials. In PC, BDE28 (35.1\%), DBDPE (82.9\%), TPP (47.9\%), C 13 (38.7\%) and $\mathrm{Cl}_{7}(37.7 \%), \mathrm{C}_{14}(69.0 \%)$ and $\mathrm{Cl}_{8}(33.5 \%)$, and $\mathrm{Zn}$ (58.3\%) were the dominant PBDEs, NBFRs, OPEs, SCCPs, MCCPs, and HM compounds, respectively. In TPU and SG, DBDPE, TPP, $\mathrm{Cl}_{7}, \mathrm{C}_{14}$, and $\mathrm{Cl}_{8}$, and $\mathrm{Zn}$ were also dominant monomers, the proportions of which were similar to those in PC. Of the other PTSs, BDE209 was predominant in TPU and SG, accounting for 24.5 and $43.0 \%$, respectively. The proportions of TPP in TPU and SG were 84.1 and $86.4 \%$, which were much higher than those in PC. $\mathrm{C}_{13}(44.1 \%)$ and $\mathrm{C}_{10}(35.4 \%)$ were predominant in TPU and SG. One-way ANOVA indicated significant differences $(P<0.05)$ in the proportions of BDE28, BDE47, BDE209, TBPH, TPP, $\mathrm{C}_{13}$, Se, $\mathrm{Cd}$, and $\mathrm{Pb}$ between PC and SG (Table S10). Next, the compositions of PTSs were compared between CPCMPs and UPCMPs (Figure S2). DBDPE, TPP, $\mathrm{Zn}$, and $\mathrm{C}_{14}$ were the dominant NBFRs, OPEs, HMs, and MCCPs, respectively, in all CPCMP and UPCMP materials. In PC, BDE28 and $\mathrm{C}_{13}$ were the main components in CPCMPs (43.1 and 42.5\%), while BDE209 and $\mathrm{C}_{10}$ were the main components in UPCMPs (36.2 and 32.8\%). Regarding TPU, the PTS 


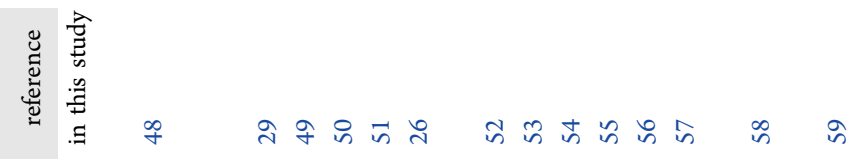

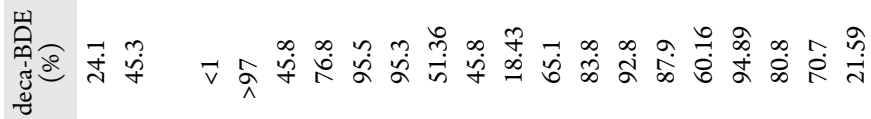

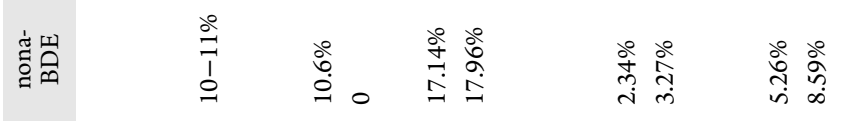

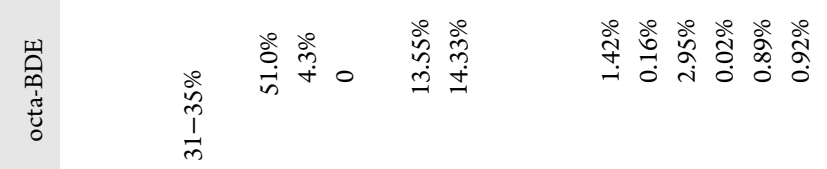

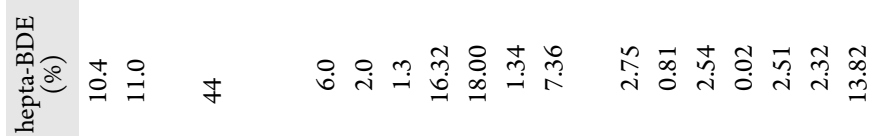

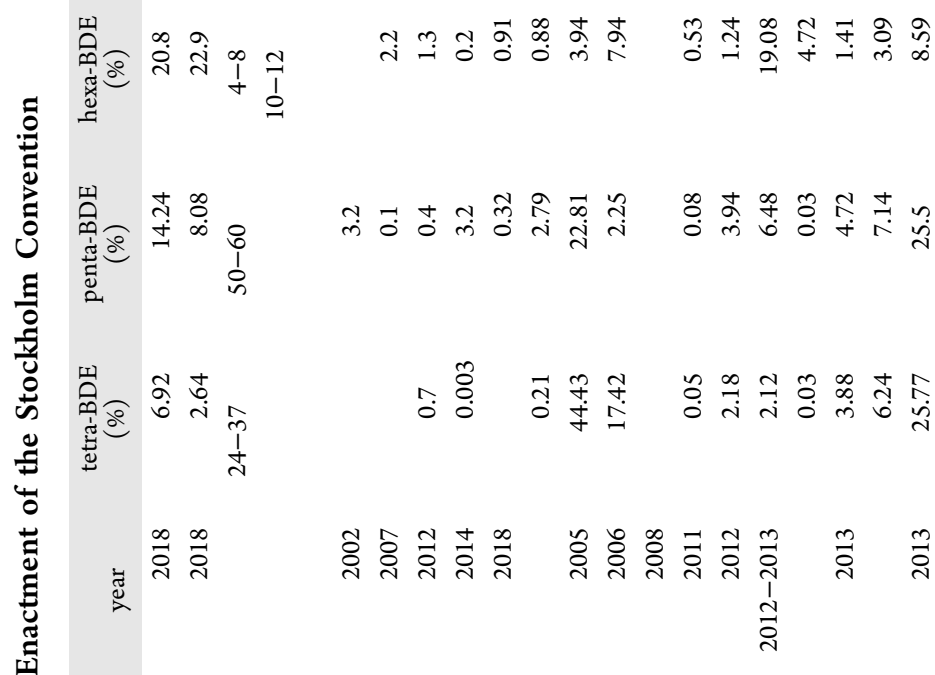

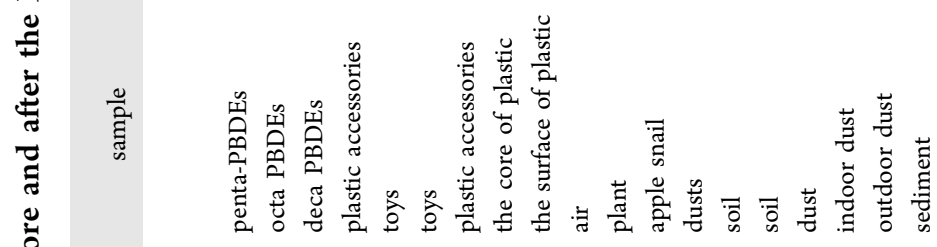

空

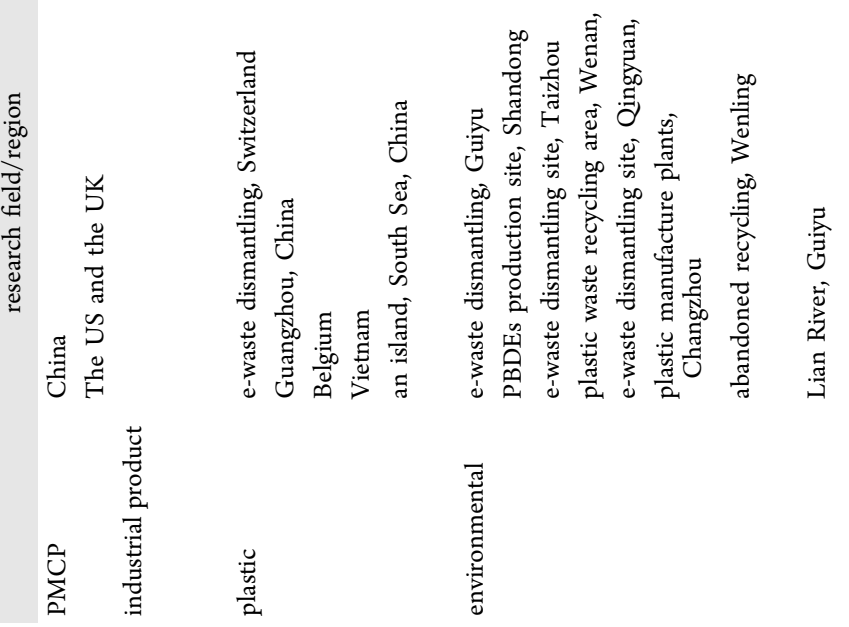


compositions varied greatly between CPCMP and UPCMP materials. The BDE209 content was $17.1 \%$ in CPCMPs and $68.9 \%$ in UPCMPs. $\mathrm{C}_{10}(46.7 \%)$ and $\mathrm{C}_{13}(37.5 \%)$ were the predominant components of CPCMP and UPCMP materials, respectively. Regarding SG, BDE209 and $\mathrm{C}_{10}$ constituted 42.6 and $44.7 \%$ of CPCMPs and 36.3 and $31.3 \%$ of UPCMPs, respectively. Among SCCPs and MCCPs, three materials had essentially the same chlorine abundance in CPCMPs and UPCMPs (see Figure S3). A one-way ANOVA comparing the different materials between CPCMPs and UPCMPs was performed (Table S9). The PTS compositions of CPCMPs differed markedly between $\mathrm{PC}$ and the other two materials, especially in terms of BDE28, TCPP, TBEP, TPP, $\mathrm{C}_{10}, \mathrm{C}_{13}$, Se, and $\mathrm{Cd}$. In UPCMPs, there were no significant differences in the compositions of the different materials, which might have been due to the small sample size.

PTS Pollution Characteristics of Unused and Used CPCMPs. The PTS concentrations were compared between unused and used CPCMPs, as shown in Figure S4. The mean PBDE, NBFR, SCCP, MCCP, and HM concentrations in PC were higher in unused than in used CPCMPs, whereas the OPE concentration did not differ significantly. In TPU and SG, the concentrations of six types of pollutants were higher in used than unused CPCMPs, except for HMs in SG; used CPCMPs were slightly lower than unused CPCMPs. There were significant $(P<0.05)$ differences in the concentrations of NBFRs, SCCPs, and MCCPs in TPU between unused and used CPCMPs and in the concentrations of PBDEs, NBFRs, OPEs, SCCPs, and MCCPs in SG between unused and used CPCMPs (Table S10). SG has been reported to have strong adsorption due to its loose porous structure and thus has been used to make wristbands as passive samplers for adsorbing BFRs. ${ }^{31,32}$ Other studies have also found that microplastics can adsorb PBDEs and HMs and accumulate pollutants. ${ }^{33,34}$ In a previous study, the BFR concentration was higher in the shells of old TV sets than in those of new ones, ${ }^{35}$ which is consistent with our results. Therefore, TPU and SG readily adsorbed PTSs. According to comparisons of the PTS compositions between unused and used CPCMPs (Figure S5), there were significant differences in $\mathrm{HBB}, \mathrm{TBEP}, \mathrm{C}_{12}$, and $\mathrm{C}_{15}$ in $\mathrm{PC}$, large differences in BDE153, BDE209, HBB, TBPH, DBDPE, TCPP, TDCPP, TCP, $\mathrm{C}_{12}$, and $\mathrm{Pb}$ in TPU, and dramatic differences in BDE99, BDE153, TCPP, TDCPP, TCP, and $\mathrm{C}_{12}$ in SG (Table S10).

Variation of Pollution Characteristics Caused by Raw Materials. In 2019, the China Mobile Industry Association developed the first domestic PCMP standard, whereas European countries have not promulgated specific standards for PCMPs. In Europe, the Restriction of Hazardous Substances standard is used for the import and export of $\mathrm{PCMPs}^{5}$ and its details are listed in Table S11. However, three mainly industrial PBDE products were unexpectedly detected in PCMPs: commercial penta-BDE (commercial pentabromodiphenyl ether), octa-BDE, and deca-BDE. Their proportions in CPCMPs and UPCMPs are shown in Table 1. Octa-BDE has never been manufactured in China, and China banned the production of penta-BDE in 2004. ${ }^{36,37}$ Deca-BDE production is not explicitly prohibited or restricted in China and therefore is widely used due to its low price. ${ }^{38}$ Europe and the United States also banned penta- and octa-BDEs in $2004,{ }^{39,40}$ the Stockholm Convention classified penta- and octa-BDEs as persistent organic pollutants and prohibited their production and use internationally in $2009,{ }^{41}$ and Europe and the United
States completely prohibited deca-BDE in $2013 .^{37,38}$ Therefore, these compounds were exported to China in various ways and recycled as raw materials. ${ }^{36,37}$ We found high proportions of BDE28 and BDE153 among PTSs, which likely are introduced in the raw materials and during the production process. According to comparisons of the percentage of industrial PBDE products before and after enactment of the Stockholm Convention (Table 1), the proportions of pentaand octa-BDEs were higher before 2009 than during 20112013, whereas that of deca-BDE showed the opposite trend, accounting for more than $90 \%$ with its use as an alternative. However, the proportions of penta- and octa-BDEs increased again around 2013, reaching pre-2009 levels. A 2008 study reported that during the photolysis process, some deca-BDE was brominated exclusively into nona-BDE (nonabromodiphenyl ether) and octa-BDE, while no PBDE homologs such as BDE-47, -99, and -100 were formed. ${ }^{42}$ Therefore, the detected penta- and octa-BDEs were not debrominated from deca-BDE. Moreover, of all of the PCMP samples, in only one sample the ratio of [DBDPE/(DBDPE + BDE209)] (0.22) was less than 0.5 , while the mean fraction of the 96 samples $(0.96 \pm 0.12)$ was much higher than 0.5 . This result suggested that the use of NBFRs has exceeded that of deca-BDE. ${ }^{43,44}$ Thus, it could be inferred that although PBDEs have been replaced by the NBFRs, there may still be a possibility of recycling waste plastics as raw materials. In addition, the value of [DBDPE/ $(\mathrm{DBDPE}+\mathrm{BDE} 209)]$ in CPCMPs $(0.95 \pm 0.13)$ was relatively higher than that in UPCMPs $(0.71 \pm 0.12)$, which showed that recycled waste plastics were used less as raw materials in the production of export PCMPs.

Between 1950 and 2015, approximately 8.3 billion tons of virgin plastic were produced worldwide, generating approximately 6300 tons of plastic waste, of which approximately $9 \%$ was recycled. ${ }^{45}$ Between $_{2006}$ and 2012, China's import of plastic waste increased from 5.9 to 8.9 million tons. ${ }^{46}$ In 2017, 69.8 million tons of plastic products were produced in China, of which approximately $30 \%$ was produced from recycled materials, and approximately 17.45 million tons were thought to contain BFRs. ${ }^{13}$ In 2017, the Chinese government announced that it would ban the import of 24 types of recyclable solid waste, including plastics, ${ }^{47}$ while the domestic garbage recycling target in China will increase by more than $42 \%$ from 246 million tons in 2015 to 350 million tons in 2020. ${ }^{46}$ Accordingly, existing PCMPs that contain high PTS concentrations will inevitably be recycled as raw materials in the production of PCMPs. Consequently, more stringent specifications for PCMPs should be adopted worldwide, including the selection of raw materials, limitations in the use of additives during the production process, and handling after use.

Health Risk Assessment of PCMPs. Average Health Risks Posed by PCMPs. A survey in 2015-2016 found that $48.2 \%$ of people use smartphones for more than $5 \mathrm{~h}$ per day and $28.8 \%$ use them for $4-5 \mathrm{~h}^{60}$ According to the "2017 Smartphone Overdependence Study" released by the National Information Society, the total numbers of smartphone users are increasing annually in all age groups. ${ }^{61}$ Dependence on mobile phones is increasing, indicating that the duration of direct contact with PCMPs is also increasing. Generally, mobile phones are mostly hand-held during use, resulting in direct contact between the skin and PTS contaminants in the PCMP, allowing the PTSs to enter or adhere to the skin. As TPU and SG materials readily adsorb PTSs, PCMPs will 


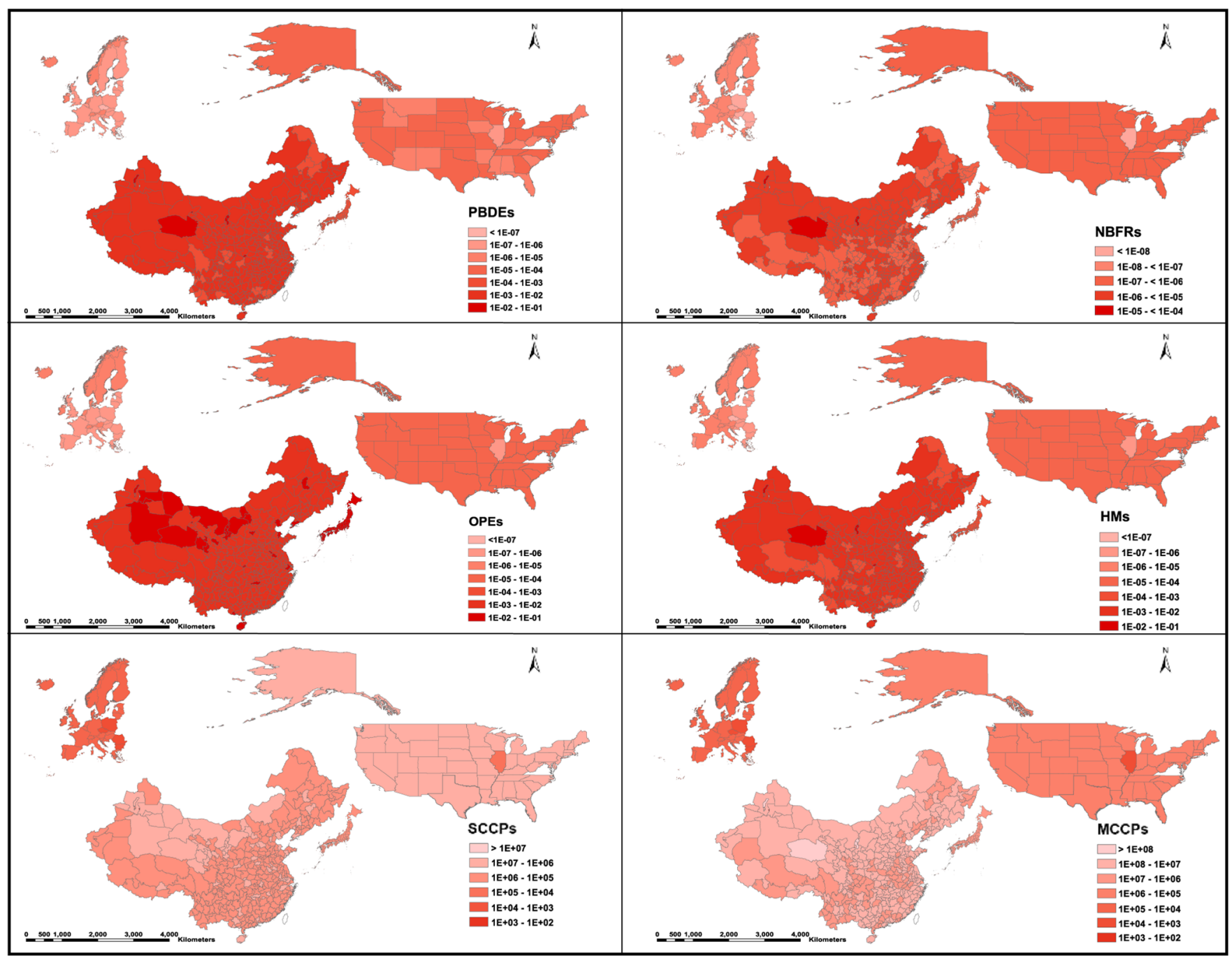

Figure 2. Spatial distribution of PTSs arising from the use of PCMPs at national and urban levels.

accumulate PTSs over time, and their impact on human health will be amplified. Placement of PCMPs in a pocket when not in use leads to gradual wear of the PCMP surface, which can ultimately lead to the ingestion of suspended pollutants. The $\mathrm{HI}$ and $\mathrm{CR}$ values were utilized to assess these health risks. Table S12 lists the hazard quotients (HQs) of different compounds that were calculated by the Monte Carlo simulation in CPCMPs and UPCMPs. The average and 95th percentile values of $\mathrm{HI}$ were less than 1 for PBDEs, NBFRs, OPEs, and HMs in all samples, and the average and 95th percentile values of $\mathrm{CR}$ of As were less than $10^{-6}$. The average and 95th percentile values of MOE of SCCPs and MCCPs were higher than 1000. These results indicated that PCMPs posed no health risks to the general and the high-exposure populations. According to the probability distribution of the HQ value of TPPs in CPCMPs (Figure S6), 80\% of the population was exposed to the maximum acceptable level of TPPs, while the 95th percentile value of TPPs of HQ in CPCMPs was 1.70 and the maximum value was 27.6. This indicated that a small number of populations may have high chronic risk due to the high concentration of TPPs in some CPCMPs.

Spatial Patterns of Health Risks from PCMPs. PCMP production is increasing globally, and China contributes $32.6 \%$ of the global production. The number of domestic sales of PCMPs made in China reached 6.17 million in 2018. The amounts of PTSs generated by consumption in China were $70.1 \mathrm{~g}$ of PBDEs, $1.13 \mathrm{~kg}$ of NBFRs, $78.9 \mathrm{~kg}$ of OPEs, $33 \mathrm{~kg}$ of SCCPs, $28.6 \mathrm{~kg}$ of MCCPs, and $625 \mathrm{~kg}$ of HMs. Due to economic globalization, PCMPs made in China have increasingly been exported to other countries, with the number reaching 134 million in 2018. The United States, Europe, and Japan accounted for 15.6, 24.3, and 5.6\% of China's PCMP exports in 2018, respectively. ${ }^{7}$ This means that China exported $9.01 \mathrm{~g}$ of PBDEs, $551 \mathrm{~g}$ of NBFRs, $9.34 \mathrm{~kg}$ of OPEs, $410 \mathrm{~g}$ of SCCPs, $387 \mathrm{~g}$ of MCCPs, and $296 \mathrm{~kg}$ of HMs via trade in PCMPs in 2018. Figure 2 and Table S13 show the distribution of PTSs in China and its export regions. The maps were from Arcgis 10.2. ${ }^{62}$ The spatial distributions of HI and CR refer mainly to the risks to human health in different regions due to PCMP trade in 2018. The average HQ value of the compounds in the PCMP was used to assess the health risks in different regions. The results are listed in Table S13 and Figure 3. In all regions, the HI values of PBDEs, NBFRs, OPEs, and HMs were less than 1 , the $\mathrm{CR}$ was less than $10^{-6}$, and the MOE of SCCPs exceeded 1000, which indicated that there was no risk to health for most people. The MOEs of MCCPs in Bulgaria, Czechia, Romania, and Poland were less 


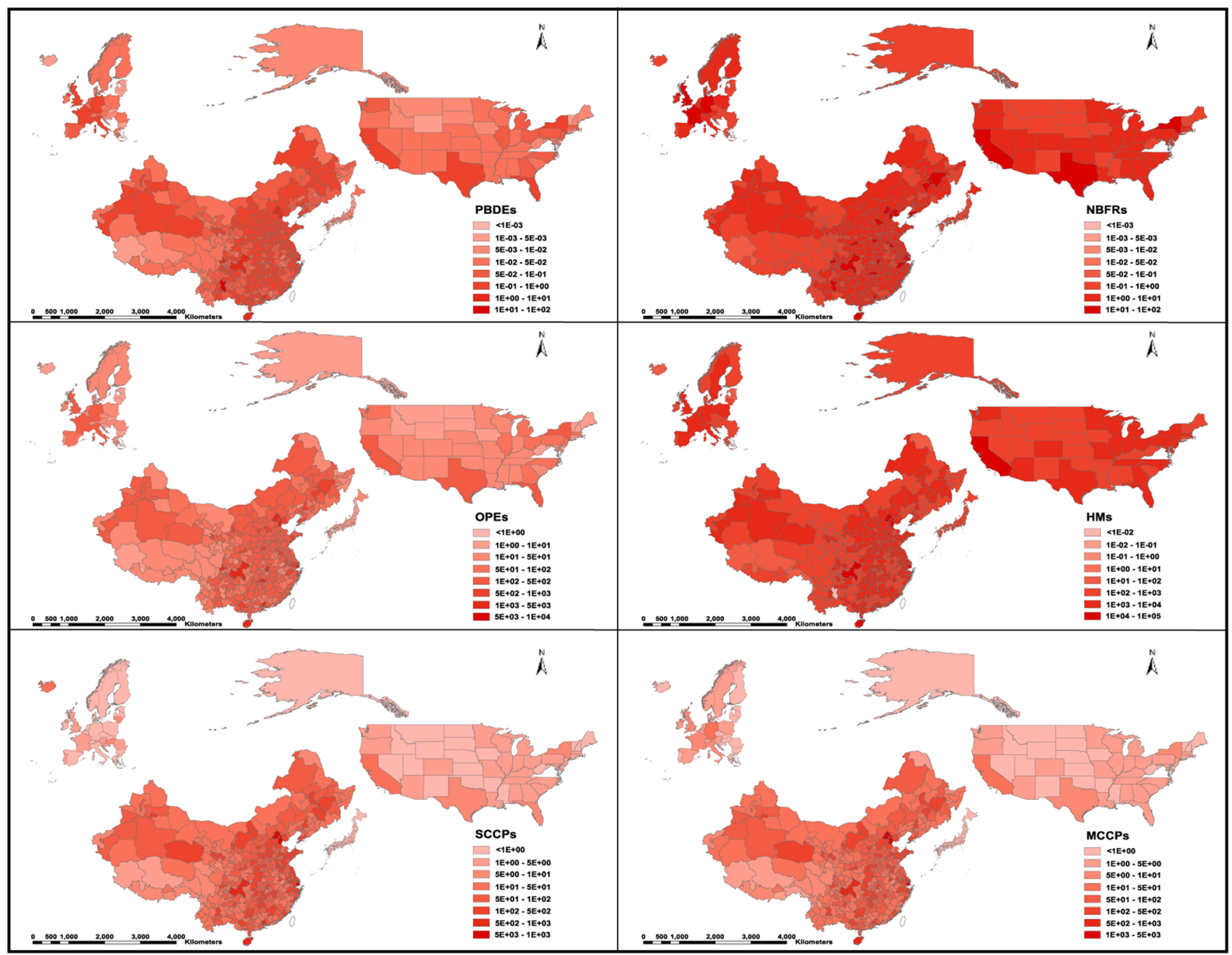

Figure 3. Spatial distribution of health risks arising from the use of PCMPs at national and urban levels.

than 1000 , which posed significant health risks to local people. China is the main producer of PCMPs and has become both an exporter and importer with economic globalization. In recent years, China's import and export of PCMPs have increased (Figure S7). The level of PTSs introduced via import trade has not been calculated. For exporting countries, the sources of other local PCMPs have not been assessed, but the distribution of pollutants and their impact on human health are likely greater than those we found in this study and are worthy of attention.

Implications. This study showed that PCMPs contain high levels of PTSs. Direct skin contact arising from frequent contact with PCMPs would not adversely affect the health of most of the general population. In addition, the PTSs contained in or adsorbed on PCMP surfaces are likely to be ingested inadvertently by touching food and the mouth with hands that have been holding a PCMP. Therefore, based on the experimental basis of this work, we hope to conduct additional research on the human risk of ingestion of PTSs from PCMPs and to investigate the relationship between the efficiency of PCMP adsorption of PTSs and the impact on human health.

The concentrations and compositions of PTSs differed markedly among the samples because there are no unified systematic standards for the raw materials and processing technology of PCMPs. From the perspective of human health, TPU and SG can absorb PTSs and thus may not be suitable as raw materials for PCMPs. Although China has set standards for PCMPs, the Restriction of Hazardous Substances implemented in Europe and the United States set a range only for the total amount of PTSs in PCMPs. We recommend formulation of specific PCMP standards globally, including the selection of raw materials, production processes, and their recycling and disposal after use, which could reduce the PTS levels in PCMPs and protect human health.

\section{ASSOCIATED CONTENT}

\section{SI Supporting Information}

The Supporting Information is available free of charge at https://pubs.acs.org/doi/10.1021/acs.est.0c07603.

Quality assurance and quality control; explanations and parameters of health risk assessment methods; full names and abbreviations of compounds of PTSs in this study (Table $S 1$ ); recovery rate and method detection limits (MDLs) for targeted PTSs (Table S2); recoveries in all spiked samples (Table S3); parameters and input assumptions for the health risk assessment (Table S4); absorption factor of different compounds (Table S5); reference dose (RfD) values for 
targeted PTSs (Table S6); concentration distribution and detection rate of various compounds (Table S7); results of a paired $t$ test of concentration and one-way ANOVA of the composition of different materials of PCMPs (Table S8); results of a paired $t$ test of concentration and one-way ANOVA of composition for different materials of CPCMPs and UPCMPs (Table S9); results of a paired $t$ test of concentration and oneway ANOVA of composition for unused and used CPCMPs (Table S10); Restriction of Hazardous Substances limits (Table S11); HQ of different compounds in CPCMPs and UPCMPs (Table S12); spatial distribution of PTSs (g) and health risk assessment results of PCMP consumption across China and major exporting areas in 2018 (Table S13); concentrations of PTSs in different materials of CPCMPs and UPCMPs (Figure S1); composition (mass fraction/\%) of PTSs in different materials of CPCMPs and UPCMPs (Figure S2); distribution (mass fraction/\%) of chlorine abundance in SCCPs and MCCPs in different materials of CPCMPs and UPCMPs (Figure S3); concentrations of PTSs in different materials of unused and used CPCMPs (Figure S4); composition (mass fraction/\%) of PTSs in different materials of CPCMPs (Figure S5); probability distribution of HQ of TPP in CPCMPs (Figure S6); and production, domestic consumption, export, and import (100 million/year) of PCMPs in China in 2014-2018 (Figure S7) (PDF)

\section{AUTHOR INFORMATION}

\section{Corresponding Author}

Qilu Li - Key Laboratory for Yellow River and Huai River Water Environment and Pollution Control, Ministry of Education, Henan Key Laboratory for Environmental Pollution Control, School of Environment, Henan Normal University, Xinxiang, Henan 453007, China; 10 orcid.org/ 0000-0001-6058-9353; Phone: (86) 3733325 971; Email: lqlblue@hotmail.com

\section{Authors}

Meng Yuan - Key Laboratory for Yellow River and Huai River Water Environment and Pollution Control, Ministry of Education, Henan Key Laboratory for Environmental Pollution Control, School of Environment, Henan Normal University, Xinxiang, Henan 453007, China

Jingfang Shangguan - School of Pharmacy, Xinxiang Medical University, Xinxiang, Henan 453003, China

Mengran Guo - Key Laboratory for Yellow River and Huai River Water Environment and Pollution Control, Ministry of Education, Henan Key Laboratory for Environmental Pollution Control, School of Environment, Henan Normal University, Xinxiang, Henan 453007, China

Jiajia Wu - Agilent Technologies (China), Inc., Beijing 100102, China

Ying Zhang - Key Laboratory for Yellow River and Huai River Water Environment and Pollution Control, Ministry of Education, Henan Key Laboratory for Environmental Pollution Control, School of Environment, Henan Normal University, Xinxiang, Henan 453007, China

Mengyuan Pei - Key Laboratory for Yellow River and Huai River Water Environment and Pollution Control, Ministry of Education, Henan Key Laboratory for Environmental
Pollution Control, School of Environment, Henan Normal University, Xinxiang, Henan 453007, China

Ziyi Zhang - Key Laboratory for Yellow River and Huai River Water Environment and Pollution Control, Ministry of Education, Henan Key Laboratory for Environmental Pollution Control, School of Environment, Henan Normal University, Xinxiang, Henan 453007, China

Mengqiong Zhang - Key Laboratory for Yellow River and Huai River Water Environment and Pollution Control, Ministry of Education, Henan Key Laboratory for Environmental Pollution Control, School of Environment, Henan Normal University, Xinxiang, Henan 453007, China

Yujing Sun - Key Laboratory for Yellow River and Huai River Water Environment and Pollution Control, Ministry of Education, Henan Key Laboratory for Environmental Pollution Control, School of Environment, Henan Normal University, Xinxiang, Henan 453007, China

Chongguo Tian - Key Laboratory of Coastal Environmental Processes and Ecological Remediation, Yantai Institute of Coastal Zone Research, Chinese Academy of Sciences, Yantai 264003, China

Jun Li - State Key Laboratory of Organic Geochemistry, Guangzhou Institute of Geochemistry, Chinese Academy of Sciences, Guangzhou 510640, China; 이이.org/00000002-3637-1642

Gan Zhang - State Key Laboratory of Organic Geochemistry, Guangzhou Institute of Geochemistry, Chinese Academy of Sciences, Guangzhou 510640, China; ㅈorcid.org/00000002-9010-8140

Complete contact information is available at: https://pubs.acs.org/10.1021/acs.est.0c07603

\section{Notes}

The authors declare no competing financial interest.

\section{ACKNOWLEDGMENTS}

This study was jointly supported by the National Natural Science Foundation (41703126) and the China Postdoctoral Science Foundation (2018T110727). Thanks are due to Jianbo Zhai, Yanqiang Wang, and Shuyang Li for their help with this experiment during sample preparation.

\section{REFERENCES}

(1) Singh, N.; Li, J. H.; Zeng, X. L. Global responses for recycling waste CRTs in e-waste. Waste Manage. 2016, 57, 187-197.

(2) Tansel, B. From electronic consumer products to e-wastes: Global outlook, waste quantities, recycling challenges. Environ. Int. 2017, 98, 35-45.

(3) Andrews, S.; Ellis, D. A.; Shaw, H.; Piwek, L. Beyond SelfReport: Tools to Compare Estimated and Real-World Smartphone Use. PLoS One 2015, 10, No. e0139004.

(4) Zong, W. Y.; Zhang, J. Y. Use of smartphone applications and its impacts on urban life: A survey and random forest analysis in Japan. Sustainable Cities Soc. 2019, 49, No. 101589.

(5) Zhang, J. H.; Gao, J. Interpretation of the Group Standard of "General Specification for Protective Case of Mobile Phone". Standard Attention 2019, 03, 9-11.

(6) Velmurugan, M. S. Sustainable perspectives on energy consumption, EMRF, environment, health and accident risks associated with the use of mobile phones. Renewable Sustainable Energy Rev. 2017, 67, 192-206.

(7) Beijing Zhongjing Vision Information Consulting Co., L., Indepth analysis and prospects of China's Protective Case of Mobile Phone market, 2019. 
(8) Pivnenko, K.; Granby, K.; Eriksson, E.; Astrup, T. F. Recycling of plastic waste: Screening for brominated flame retardants (BFRs). Waste Manage. 2017, 69, 101-109.

(9) Kuang, J. M.; Abdallah, M. A.-E.; Harrad, S. Brominated flame retardants in black plastic kitchen utensils: Concentrations and human exposure implications. Sci. Total Environ. 2018, 610-611, 11381146.

(10) Li, T. Y.; Bao, L. J.; Wu, C. C.; Liu, L. Y.; Wong, C. S.; Zeng, E. Y. Organophosphate flame retardants emitted from thermal treatment and open burning of e-waste. J. Hazard. Mater. 2019, 367, 390-396.

(11) Cao, D. D.; Gao, W.; Wu, J.; Lv, K.; Xin, S. Z.; Wang, Y. W.; Jiang, G. B. Occurrence and Human Exposure Assessment of Shortand Medium-Chain Chlorinated Paraffins in Dusts from Plastic Sports Courts and Synthetic Turf in Beijing, China. Environ. Sci. Technol. 2019, 53, 443-451.

(12) Tang, Z. W.; Zhang, L. Z.; Huang, Q. F.; Yang, Y. F.; Nie, Z. Q.; Cheng, J. L.; Yang, J.; Wang, Y. W.; Chai, M. Contamination and risk of heavy metals in soils and sediments from a typical plastic waste recycling area in North China. Ecotoxicol. Environ. Saf. 2015, 122, 343-351.

(13) Li, Y.; Chang, Q.; Duan, H. B.; Liu, Y. C.; Zhang, J.; Li, J. H. Occurrence, levels and profiles of brominated flame retardants in daily-use consumer products on the Chinese market. Environ. Sci.: Processes Impacts 2019, 21, 446-455.

(14) Wei, G.-L.; Li, D. Q.; Zhuo, M. N.; Liao, Y. S.; Xie, Z. Y.; Guo, T. L.; Li, J. J.; Zhang, S. Y.; Liang, Z. Q. Organophosphorus flame retardants and plasticizers: Sources, occurrence, toxicity and human exposure. Environ. Pollut. 2015, 196, 29-46.

(15) Xu, C.; Gao, L. R.; Zheng, M. H.; Qiao, L.; Cui, L. L.; Wang, K. R.; Huang, D. Short- and medium-chain chlorinated paraffins in commercial rubber track products and raw materials. J. Hazard. Mater. 2019, 380, No. 120854.

(16) van der Veen, I.; de Boer, J. Phosphorus flame retardants: Properties, production, environmental occurrence, toxicity and analysis. Chemosphere. 2012, 88, 1119-1153.

(17) Burges, A.; Epelde, L.; Garbisu, C. Impact of repeated singlemetal and multi-metal pollution events on soil quality. Chemosphere. 2015, 120, 8-15.

(18) Guney, M.; Zagury, G. J.; Dogan, N.; Onay, T. T. Exposure assessment and risk characterization from trace elements following soil ingestion by children exposed to playgrounds, parks and picnic areas. J. Hazard. Mater. 2010, 182, 656-664.

(19) Wang, Y.; Wang, Y.; Lee, S. H. The Effect of Cross-Border ECommerce on China's International Trade: An Empirical Study Based on Transaction Cost Analysis. Sustainability. 2017, 9, 2028.

(20) Chen, S. J.; Ma, Y. J.; Wang, J.; Chen, D.; Luo, X. J.; Mai, B. X. Brominated Flame Retardants in Children's Toys: Concentration, Composition, and Children's Exposure and Risk Assessment. Environ. Sci. Technol. 2009, 43, 4200-4206.

(21) Wang, C.; Gao, W.; Liang, Y.; Wang, Y. W.; Jiang, G. B. Concentrations and congener profiles of chlorinated paraffins in domestic polymeric products in China. Environ. Pollut. 2018, 238, $326-335$.

(22) Wan, W. N.; Zhang, S. Z.; Huang, H. L.; Wu, T. Occurrence and distribution of organophosphorus esters in soils and wheat plants in a plastic waste treatment area in China. Environ. Pollut. 2016, 214, 349-353.

(23) Liu, X. T.; Yu, G.; Cao, Z. G.; Wang, B.; Huang, J.; Deng, S. B.; Wang, Y. J. Occurrence of organophosphorus flame retardants on skin wipes: Insight into human exposure from dermal absorption. Environ. Int. 2017, 98, 113-119.

(24) U.S. EPA. Dermal Exposure Assessment: A Summary of EPA Approaches. 2007, https://search.epa.gov/epasearch/?querytext= Dermal+Exposure + Assessment $\% 3 \mathrm{~A}+\mathrm{A}+$ Summary + of $+\mathrm{EPA}+$ Approa ches \&areaname $=\&$ areacontacts $=\&$ areasearchurl $=\&$ typeofsearch $=$ epa\&result template $=2 \mathrm{col} . \mathrm{ftl} \# /$.

(25) Cao, D. D.; Gao, W.; Wu, J.; Lv, K.; Xin, S. Z.; Wang, Y. W.; Jiang, G. B. Occurrence and Human Exposure Assessment of Shortand Medium-Chain Chlorinated Paraffins in Dusts from Plastic Sports
Courts and Synthetic Turf in Beijing, China. Environ. Sci. Technol. 2019, 53, 443-451.

(26) Li, Z. Y.; Ma, Z. W.; van der Kuijp, T. J.; Yuan, Z. W.; Huang, L. A review of soil heavy metal pollution from mines in China: Pollution and health risk assessment. Sci. Total Environ. 2014, 468469, 843-853.

(27) Pieters, M. N.; Kramer, H. J.; Slob, W. Evaluation of the uncertainty factor for subchronic-to-chronic extrapolation: statistical analysis of toxicity data. Regul. Toxicol. Pharmacol. 1998, 27, 108-11.

(28) Zhou, Q.; Yang, J. X.; Liu, M. M.; Liu, Y.; Sarnat, S.; Bi, J. Toxicological Risk by Inhalation Exposure of Air Pollution Emitted from China's Municipal Solid Waste Incineration. Environ. Sci. Technol. 2018, 52, 11490-11499.

(29) Cao, X. P.; Wang, L.; Zhang, Y.; Li, Y.; Zhu, C. Y.; Zheng, X. B.; Sun, Y. X.; Li, D. N.; Mo, L.; Mai, B. X. Occurrence of organic pollutants in plastics on beach: Stranded foams can be sources of pollutants in islands. Sci. Total Environ. 2020, 707, No. 136119.

(30) Wang, Y.; Hou, M. M.; Zhang, Q. N.; Wu, X. W.; Zhao, H. X.; Xie, Q.; Chen, J. W. Organophosphorus Flame Retardants and Plasticizers in Building and Decoration Materials and Their Potential Burdens in Newly Decorated Houses in China. Environ. Sci. Technol. 2017, 51, 10991-10999.

(31) O'Connell, S. G.; Kind, L. D.; Anderson, K. A. Silicone Wristbands as Personal Passive Samplers. Environ. Sci. Technol. 2014, 48, 3327-3335.

(32) Hammel, S. C.; Phillips, A. L.; Hoffman, K.; Stapleton, H. M. Evaluating the Use of Silicone Wristbands to Measure Personal Exposure to Brominated Flame Retardants. Environ. Sci. Technol. 2018, 52, 11875-11885

(33) Xu, P. C.; Ge, W.; Chai, C.; Zhang, Y.; Jiang, T.; Xia, B. Sorption of polybrominated diphenyl ethers by microplastics. Mar. Pollut. Bull. 2019, 145, 260-269.

(34) Turner, A. Heavy metals, metalloids and other hazardous elements in marine plastic litter. Mar. Pollut. Bull. 2016, 111, 136142.

(35) Morf, L. S.; Tremp, J.; Gloor, R.; Huber, Y.; Stengele, M.; Zennegg, M. Brominated flame retardants in waste electrical and electronic equipment: substance flows in a recycling plant. Environ. Sci. Technol. 2005, 39, 8691-8699.

(36) Shaw, S. D.; Blum, A.; Weber, R.; Kannan, K.; Rich, D.; Lucas, D.; Koshland, C. P.; Dobraca, D.; Hanson, S.; Birnbaum, L. S. Halogenated flame retardants: do the fire safety benefits justify the risks? Rev. Environ. Health. 2010, 25, 261-305.

(37) Ni, K.; Lu, Y. L.; Wang, T. Y.; Shi, Y. J.; Kannan, K.; Xu, L.; Li, Q. S.; Liu, S. J. Polybrominated diphenyl ethers (PBDEs) in China: Policies and recommendations for sound management of plastics from electronic wastes. J. Environ. Manage. 2013, 115, 114-123.

(38) Liang, S.; Xu, F.; Tang, W. B.; Zhang, Z.; Zhang, W.; Liu, L. L.; Wang, J. X.; Lin, K. F. Brominated flame retardants in the hair and serum samples from an e-waste recycling area in southeastern China: the possibility of using hair for biomonitoring. Environ. Sci. Pollut. Res. 2016, 23, 14889-14897.

(39) Möller, A.; Xie, Z. Y.; Cai, M. H.; Zhong, G. C.; Huang, P.; Cai, M. G.; Sturm, R.; He, J. F.; Ebinghaus, R. Polybrominated Diphenyl Ethers vs Alternate Brominated Flame Retardants and Dechloranes from East Asia to the Arctic. Environ. Sci. Technol. 2011, 45, 67936799.

(40) Lee, S. Y.; Song, G. J.; Kannan, K.; Moon, H. B. Occurrence of PBDEs and other alternative brominated flame retardants in sludge from wastewater treatment plants in Korea. Sci. Total Environ. 2014 470-471, 1422-1429.

(41) Zheng, Q.; Nizzetto, L.; Li, J.; Mulder, M. D.; Sanka, O.; Lammel, G.; Bing, H. J.; Liu, X.; Jiang, Y. S.; Luo, C. L.; Zhang, G. Spatial Distribution of Old and Emerging Flame Retardants in Chinese Forest Soils: Sources, Trends and Processes. Environ. Sci. Technol. 2015, 49, 2904-2911.

(42) Kajiwara, N.; Noma, Y.; Takigami, H. Photolysis studies of technical decabromodiphenyl ether (DecaBDE) and ethane (DeBD- 
ethane) in plastics under natural sunlight. Environ. Sci. Technol. 2008, 42, 4404-4409.

(43) Ma, Y. N.; Salamova, A.; Venier, M.; Hites, R. A. Has the Phase-Out of PBDEs Affected Their Atmospheric Levels? Trends of PBDEs and Their Replacements in the Great Lakes Atmosphere. Environ. Sci. Technol. 2013, 47, 11457-11464.

(44) Chen, S.-J.; Feng, A. H.; He, M. J.; Chen, M. Y.; Luo, X. J.; Mai, B. X. Current Levels and Composition Profiles of PBDEs and Alternative Flame Retardants in Surface Sediments from the Pearl River Delta, Southern China: Comparison with Historical Data. Sci. Total Environ. 2013, 444, 205-211.

(45) Babayemi, J. O.; Nnorom, I. C.; Osibanjo, O.; Weber, R. Ensuring sustainability in plastics use in Africa: consumption, waste generation, and projections. Environ. Sci. Eur. 2019, 31, No. 60.

(46) Liu, Z.; Adams, M.; Walker, T. R. Are exports of recyclables from developed to developing countries waste pollution transfer or part of the global circular economy? Resour., Conserv. Recycl. 2018, $136,22-23$.

(47) Walker, T. R. China's ban on imported plastic waste could be a game changer. Nature 2018, 553, 405.

(48) Guerra, P. A. M.; Eljarrat, E.; Barceló, D. Introduction to Brominated Flame Retardants: Commercially Products, Applications, and Physicochemical Properties. In The Handbook of Environmental Chemistry; Springer, 2010; Vol. 16, p 93.

(49) Chen, S. J.; Ma, Y. J.; Wang, J.; Chen, D.; Luo, X. J.; Mai, B. X. Brominated Flame Retardants in Children's Toys: Concentration, Composition, and Children's Exposure and Risk Assessment. Environ. Sci. Technol. 2010, 44, 1156.

(50) Ionas, A. C.; Dirtu, A. C.; Anthonissen, T.; Neels, H.; Covaci, A. Downsides of the recycling process: Harmful organic chemicals in children's toys. Environ. Int. 2014, 65, 54-62.

(51) Anh, H. Q.; Nam, V. D.; Tri, T. M.; Ha, N. M.; Ngoc, N. T.; Mai, P. T. N.; Anh, D. H.; Minh, N. H.; Tuan, N. A.; Minh, T. B. Polybrominated diphenyl ethers in plastic products, indoor dust, sediment and fish from informal e-waste recycling sites in Vietnam: a comprehensive assessment of contamination, accumulation pattern, emissions, and human exposure. Environ. Geochem. Health 2017, 39, 935-954.

(52) Chen, D. H.; Bi, X. H.; Zhao, J. P.; Chen, L. G.; Tan, J. H.; Mai, B. X.; Sheng, G. Y.; Fu, J. M.; Wong, M. H. Pollution characterization and diurnal variation of PBDEs in the atmosphere of an E-waste dismantling region. Environ. Pollut. 2009, 157, 1051-1057.

(53) Jin, J.; Liu, W. Z.; Wang, Y.; Tang, X. Y. Levels and distribution of polybrominated diphenyl ethers in plant, shellfish and sediment samples from Laizhou Bay in China. Chemosphere 2008, 71, 10431050.

(54) Fu, J. J.; Wang, Y. W.; Zhang, A. Q.; Zhang, Q. H.; Zhao, Z. S.; Wang, T.; Jiang, G. B. Spatial distribution of polychlorinated biphenyls (PCBs) and polybrominated biphenyl ethers (PBDEs) in an e-waste dismantling region in Southeast China: Use of apple snail (Ampullariidae) as a bioindicator. Chemosphere 2011, 82, 648-655.

(55) Tang, Z. W.; Huang, Q. F.; Yang, Y. F.; Nie, Z. Q.; Cheng, J. L.; Yang, J.; Wang, Y. W.; Chai, M. Polybrominated diphenyl ethers (PBDEs) and heavy metals in road dusts from a plastic waste recycling area in north China: implications for human health. Environ. Sci. Pollut. Res. 2016, 23, 625-637.

(56) Wang, J. X.; Liu, L. L.; Wang, J. F.; Pan, B. S.; Fu, X. X.; Zhang, G.; Zhang, L.; Lin, K. F. Distribution of metals and brominated flame retardants (BFRs) in sediments, soils and plants from an informal ewaste dismantling site, South China. Environ. Sci. Pollut. Res. 2015, 22, 1020-1033.

(57) Xu, J.; Qian, W. Y.; Li, J. Y.; Zhang, X. F.; He, J.; Kong, D. Y. Polybrominated diphenyl ethers (PBDEs) in soil and dust from plastic production and surrounding areas in eastern of China. Environ. Geochem. Health. 2019, 41, 2315-2327.

(58) Xu, F.; Liu, Y. C.; Wang, J. X.; Zhang, G.; Zhang, W.; Liu, L. L.; Wang, J. F.; Pan, B. S.; Lin, K. F. Characterization of heavy metals and brominated flame retardants in the indoor and outdoor dust of e- waste workshops: implication for on-site human exposure. Environ. Sci. Pollut. Res. 2015, 22, 5469-5480.

(59) Li, H. R.; La Guardia, M. J.; Liu, H. H.; Hale, R. C.; Mainor, T. M.; Harvey, E.; Sheng, G. Y.; Fu, J. M.; Peng, P. A. Brominated and organophosphate flame retardants along a sediment transect encompassing the Guiyu, China e-waste recycling zone. Sci. Total. Environ. 2019, 646, 58-67.

(60) Jia, H. J.; Ma, A. D.; Wang, H. X. Statistical analysis of people using mobile phones. Technology Wind 2019, 23, 241.

(61) Kim, J. S.; Lim, C. A. Proposal of Interactive Contents for Prevention of Smartphone Overdependence. Int. J. Advanced. Culture. Technol. 2019, 7, 70-76.

(62) Arcmap. 10.2. https://services7.arcgis.com/ AUkh0eiMhEaQlmEh/arcgis/rest/services/chn_cities/FeaureServer. https://services.arcgis.com/JmSyAMvJJkgxqun6/arcgis/rest/ services/healthy_life_years_Europe_2008_2017/FeatureServer. https://services.arcgis.com/P3ePLM MYs2 $\overline{R V C h k J x / a r c g i s / r e s t / ~}$ services/USA_States_Generalized/FeatureServer.2020. 